\title{
Lentiviral-mediated short hairpin RNA silencing of APE1 suppresses hepatocellular carcinoma proliferation and migration: A potential therapeutic target for hepatoma treatment
}

\author{
ZHI-HUA ZHENG ${ }^{1,2^{*}}$, WEI DU ${ }^{2,3^{*}}$, YAN-JU LI ${ }^{2}$, MEI-QIN GAO ${ }^{2}$, AI-MIN HUANG ${ }^{2}$ and JING-FENG LIU ${ }^{4,5}$ \\ ${ }^{1}$ Department of Pathology, Quanzhou Medical College, Quanzhou, Fujian 362100; ${ }^{2}$ Department of Pathology and \\ Institute of Oncology, Fujian Medical University, Fuzhou, Fujian 350004; ${ }^{3}$ Department of Pathology, \\ People's Hospital Affiliated to Fujian University of Traditional Chinese Medicine, Fuzhou, Fujian 350004; \\ ${ }^{4}$ Mengchao Hepatobiliary Hospital of Fujian Medical University, Fuzhou, Fujian 350025; \\ ${ }^{5}$ The Liver Center of Fujian Province, Fujian Medical University, Fuzhou, Fujian 350025, P.R. China
}

Received December 19, 2014; Accepted March 20, 2015

DOI: $10.3892 / o r .2015 .3976$

\begin{abstract}
Apurinic/apyrimidinic endonuclease-1 (APE1) is a protein involved in DNA repair and transcriptional regulation of gene expression. APE1 expression was reported to be correlated with poor prognosis in hepatocellular carcinoma (HCC) patients. Based on our previous study, we hypothesized that APE1 may be involved in the metastatic progression of HCC. Thus, the present study aimed to investigate the knockdown effect of APE1 using shRNA in HCC and demonstrate that silencing of APE1 in MHCC97-H cells can decrease the oncogenic transforming potential in vitro and reduce the growth of HCC tumor xenografts in vivo. Silencing of APE1 expression decreased the cell proliferation and survival, reduced the cell adhesion ability in Matrigel or fibronectin-coated plates and suppressed the cell migration and invasion in a Transwell assay of HCC cells. In the xenograft study, tumor growth was markedly inhibited in the APE1-silenced group. Silencing of APE1 in MHCC97-H cells decreased the oncogenic transforming potential in vitro and reduced the growth of $\mathrm{HCC}$ tumor xenografts in vivo. Inhibition of APE1 may present a novel therapeutic approach for the treatment of HCC.
\end{abstract}

Correspondence to: Professor Ai-Min Huang, Department of Pathology and Institute of Oncology, Fujian Medical University, 88 Jiaotong Road, Fuzhou, Fujian 350004, P.R. China

E-mail: aimin@fjmu.edu

Professor Jing-Feng Liu, The Liver Center of Fujian Province, Mengchao Hepatobiliary Hospital of Fujian Medical University, 312 Xihong Road, Fuzhou, Fujian 350025, P.R. China

E-mail: drjingfeng@126.com

*Contributed equally

Key words: hepatocellular carcinoma, cell migration, proliferation, APE1, metastasis

\section{Introduction}

Hepatocellular carcinoma (HCC) is the most common primary malignant tumor type observed in the human liver (1). HCC is also the third leading cause of cancer-related mortality worldwide, and it is especially prevalent in Southeast Asia and sub-Saharan Africa (2). Despite improvement in the treatment of HCC over the last couple of decades, the mortality rate is still high due to relapse and tumor metastasis $(3,4)$. Increased understanding of HCC progression at the molecular level is crucial to develop novel targeted therapies which could be promising therapeutic options for HCC patients.

Apurinic/apyrimidinic endonuclease-1 (APE1) is a protein involved in DNA repair and transcriptional regulation of gene expression (5). Several studies have shown that APE1 is overexpressed in numerous types of cancer, including prostate, non-small cell lung, colorectal, ovarian and cervical cancer, and osteosarcoma (6-11). APE1 overexpression was also found to be associated with a poor prognosis and resistance to radiation and chemotherapeutic drugs (12). Since APE1 is a vital enzyme in the DNA repair pathway induced by irradiation damage, it may have a critical role in the cancer cell response to chemotherapy and radiotherapy. While the function of DNA repair has been extensively investigated, the role of activating transcription factors mediated by APE1 has not yet been widely studied (13). Previous studies indicated that APE1 stimulated the DNA binding activity of various transcription factors including activator protein-1 (AP-1), NF- $\mathrm{kB}, \mathrm{HIF}-1 \alpha$ and p53 $(14,15)$. However, the link between gene expression and related cell behavior is not well elucidated hampering the understanding of the function of APE1 in cancer cell biology.

Recently, APE1 was reported to be significantly overexpressed in HCC tissues than that in the surrounding cirrhotic tissues $(16,17)$. In addition, we previously showed that APE1 was highly expressed in a poorly differentiated group, capsular invasion group and metastasis group (18). We also found that 
Table I. Detected genes and primer sequences.

\begin{tabular}{lll}
\hline Gene & \multicolumn{1}{c}{ Forward primer } & \multicolumn{1}{c}{ Reverse primer } \\
\hline APE1 & \multicolumn{1}{c}{ 5'-AGAGCCAGAGGCCAAGAAGAGTA-3' } & 5'-GAAGCCCATCCACATTCCAAGAG-3' \\
c-Fos & 5'-GAGAAGCCAAGACTGAGCCG-3' & 5'-CGTTGAAGCCCGAGAACATC-3' \\
c-Jun & 5'-CTCAGACAGTGCCCGAGATG-3' & 5'-GCTGCGTTAGCATGAGTTGG-3' \\
MMP-2 & 5'-AGCCTGTTCCATGAAGGCAGA-3' & 5'-CTGGCAGCATCATCCACACATAC-3' \\
GAPDH & 5'-GTGCCCAAGAATAGATGCTGAC-3' & 5'-CGGTAGGGACATGCTAAGTAGAGT-3' \\
\hline
\end{tabular}

APE1 protein is mainly expressed in the nuclei in normal liver tissues. While in malignant liver tissues, APE1 protein is expressed in the nucleus and cytoplasm. Therefore, we hypothesized that APE1 may be involved in the metastatic progression of HCC. Based on previous results (18), this study aimed to investigate the knockdown effect of APE1 using shRNA in HCC and demonstrate that silencing of APE1 in MHCC97-H cells can decrease the oncogenic transforming potential in vitro and reduce the growth of HCC tumor xenografts in vivo. It was demonstrated that inhibition of APE1 may present a novel therapeutic approach for the treatment of HCC.

\section{Materials and methods}

Cell line. The MHCC97-H cell line was obtained from the Liver Cancer Research Institute of Fudan University (Shanghai, China). The cells were cultured at $37^{\circ} \mathrm{C}$ in a humidified incubator under 5\% $\mathrm{CO}_{2}$ and grown in Dulbecco's modified Eagle's medium (DMEM; Hyclone Company, Logan, UT, USA) supplemented with $10 \%$ fetal bovine serum (FBS; PAA Laboratories $\mathrm{GmbH}$, Pasching, Austria), $100 \mathrm{U} / \mathrm{ml}$ penicillin, and $100 \mu \mathrm{g} / \mathrm{ml}$ streptomycin.

Lentiviral vectors and infection. The APE1 RNA interference lentivirus vector named LV-APE1-shRNA and LV-NC-shRNA (control) were constructed at GeneChem Technology (Shanghai, China) as previously described (19). The shRNA targeting sequencing for APE1 was: 5'-GAGACCAAATGT TCAGAGAAC-3'. The MHCC97-H cell line was infected with LV-APE1-shRNA or LV-NC-shRNA for $2 \mathrm{~h}$ and subsequently placed in fresh medium, while the uninfected cells were used as the blank control. The cells were cultured for the next $48 \mathrm{~h}$ and then harvested for western blot analysis or prepared for the following experiments.

Western blotting and RT-PCR. Western blot analysis was performed as previously described (19) using the antibodies as follows: mouse anti-APE1 (Novus Biologicals, Littleton, CO, USA), anti-c-Fos polyclonal, anti-c-Jun, anti-caspase-3 and anti-inducible matrix metalloproteinase (MMP)-2 and a monoclonal antibody against $\beta$-actin (Merck KGaA, Darmstadt, Germany). The protein concentration was analyzed as APE1/ $\beta$-actin. Image J software (National Institutes of Health, Bethesda, MD, USA) was used for densitometric analysis.

Total cell RNA was extracted from the infected cells and control cells with TRIzol (Invitrogen, Carlsbad, CA, USA) following the manufacturer's instructions. Subsequently, reverse transcription was performed using the PrimeScript RT reagent kit (Takara Biotechnology, Dalian, China). Primers for APE1, c-Fos, c-Jun, caspase-3 and MMP-2 were designed as listed in Table I. PCR reaction was performed with 30 cycles (Piko TCP9600; Thermo Scientific, Waltham, MA, USA). The gene expression levels were analyzed as APE1/GAPDH. Densitometric analysis was performed using Image $\mathbf{J}$ software.

Cell proliferation. Cell proliferation of the infected and control cells was assessed by 3-(4,5-dimethylthiazol-2-yl)-2,5-diphenyltetrazolium bromide (MTT) assay (Sigma, New York, NY, USA). Briefly, the cells were seeded at 3,000 cells/well and were cultured for $48 \mathrm{~h}$ prior to measurement. The cells were then washed with phosphate-buffered saline (PBS; Biovision, San Francisco Bay, CA, USA) and then incubated with $0.45 \mathrm{mg} / \mathrm{ml}$ MTT solution made up in serum-free media at $37^{\circ} \mathrm{C}$. Once the formation of blue formazan crystals was apparent, the MTT solution was removed. Subsequently, $100 \mu 1$ of DMSO (Sigma) was added to solubilize the blue formazan crystal, and the absorbance was read at $570 \mathrm{~nm}$.

Cell adhesion. The adhesion assay was performed as previously described (20). Briefly, a 96-well plate was pre-coated with Matrigel and fibronectin (FN) (both from BD Biosciences, New York, NY, USA), dissolved in $50 \mu 1$ of PBS, incubated for $2 \mathrm{~h}$ at $37^{\circ} \mathrm{C}$ and then blocked for non-specific sites by the addition of $1 \%$ bovine serum albumin (BSA) in $100 \mu \mathrm{l}$ of PBS/well for 30 min. Cells with $90 \%$ confluency were harvested with a brief treatment of $0.25 \%$ trypsin (Invitrogen). The cells were plated in 96-well plates at $1 \times 10^{5}$ density and cultured for $1 \mathrm{~h}$. The medium was aspirated and the cells were washed twice with PBS to remove unattached cells. The viability of the attached cells was determined by MTT assay. All experiments were performed in triplicate with 4-8 replicates/experiment.

Cell migration and invasion. Cell migration and invasion assays were performed in BioCoat Transwell chambers (Corning Costar, Tewksbury, MA, USA). While cell migration was determined with uncoated porous inserts, cell invasion was measured using a filter precoated with Matrigel. Cells were starved under a serum-free condition $24 \mathrm{~h}$ prior to experimentation. Subsequently, the cells were plated on the upper chamber with $0.2 \%$ FBS and cultured for $24 \mathrm{~h}$. Migrated and invaded cells on the inserts were fixed using $90 \%$ ethanol 
followed by staining with $0.1 \%$ crystal violet (Sigma) for counting. The numbers of migrated and invaded cells were counted over three fields per one filter for triplicate experiments.

Xenograft tumor growth. BALB/c nude mice (Shanghai Slaccas Co., Shanghai, China) (4-5 weeks old) were used in the xenograft growth study. The animal study was performed according to procedures approved by the Animal Care and Ethics Committee of Fujian Medical University. Tumor growth was measured as follows. Briefly, a volume of $0.2 \mathrm{ml}$ of $2 \times 10^{6}$ viable LV-APE1-shRNA or LV-NC-shRNA cells was subsequently injected into the right axilla of BALB/c nude mice ( $n=6$ per group). Tumor volumes were analyzed every two days by measuring the major axis (a) and the minor axis (b) by Vernier calipers. The total volume (V) was calculated by the equation: $V=1 / 6 \pi a b^{2}$. After 3 weeks, the nude mice were euthanized, and the xenografts were resected for weight measurement. The inhibitory rate of tumor growth was calculated using the following equation: Inhibitory rate $=1$ - (average weight of experimental group - average weight of control group) x $100 \%$. Subsequently, tissue samples were collected for histological and RT-PCR analyses.

Histological analysis. Histological analysis was performed as described previously (21). In brief, $5-\mu \mathrm{m}$ sections were stained with hemotoxylin and $1 \%$ eosin Y solution separately. After that, the sections were rehydrated in a graded series of ethanol solutions, and then the ethanol was extracted with xylene. Mounting medium was added and the slide was covered with a coverslip prior to observation using a microscope. Apoptosis was measured using the terminal-deoxynucleotidyl transferase dUTP nick end labeling (TUNEL) method carried out using the TUNEL apoptosis assay kit (C1091; Beyotime, Shanghai, China) according to the manufacturer's instructions. Briefly, the sections were incubated with $0.3 \% \mathrm{H}_{2} \mathrm{O}_{2}$ for $30 \mathrm{~min}$. After washing with PBS, the sections were treated with biotin-16dUTP in reaction buffer at $37^{\circ} \mathrm{C}$ for $60 \mathrm{~min}$. Subsequently, the specimens were incubated with streptavidin-peroxidase complex and stained with DAB after twice with PBS (both from Maixin, Fuzhou, China). Finally, cell morphology was observed under a light microscope (Olympus BX43; Olympus Co., Tokyo, Japan).

Statistics. Data are expressed as mean \pm SEM from a representative experiment conducted in triplicate. Results were analyzed using unpaired two-tailed Student's t-test and P-values $<0.05$ were applied to determine statistically significance by comparing data sets.

\section{Results}

shRNA silencing of APE1 expression effectively reduces APE1 $m R N A$ and protein expression in the MHCC97-H cells. MHCC97-H is a highly metastatic HCC cell line, which expresses high endogenous APE1 according to our previous results. In this study, MHCC97-H cells were infected by a lentivirus which contained APE1 shRNA (LV-APE1-shRNA) or an empty vector (LV-NC-shRNA). Following infection, the cells were collected for mRNA and protein expression analysis
A

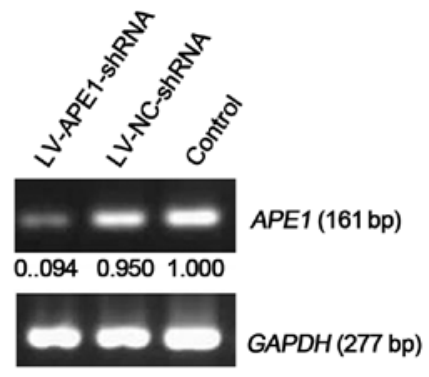

B

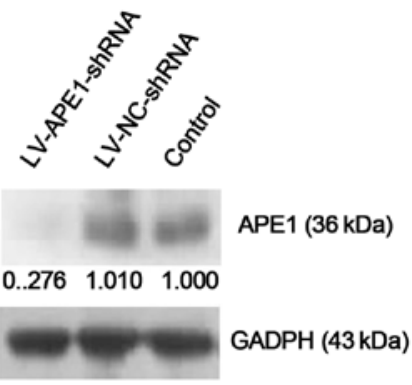

C

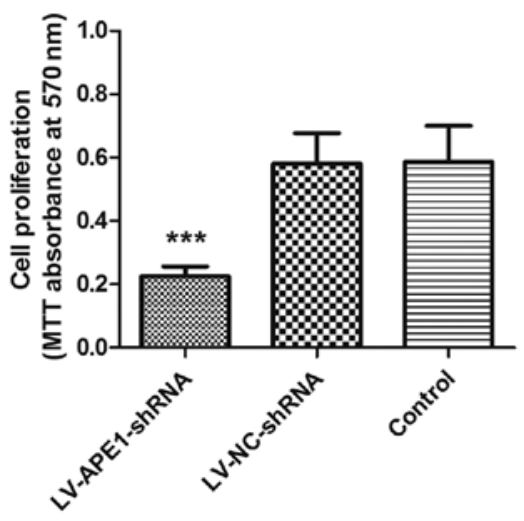

Figure 1. Silencing of APE1 decreases the proliferation of MHCC97-H cells. (A) mRNA and (B) protein expression of APE1 in the LV-APE1-shRNA, LV-NC-shRNA and control cells.(C)Cell proliferation rate of LV-APE1-shRNA, LV-NC-shRNA and control cells. ${ }^{* * *} \mathrm{P}<0.001$.

by RT-PCR and western blotting. Uninfected MHCC97-H cells were included as the negative control. Compared with the LV-NC-shRNA cells and uninfected negative control cells, the mRNA and protein expression levels of APE1 in the LV-APE1-shRNA cells was decreased. Based on the RT-PCR results, the mRNA level of APE1 was reduced by $90.6 \%$ in the LV-APE1-shRNA cells, while the protein level of APE1 was significantly reduced by $72.4 \%$ in the LV-APE1-shRNA cells vs. that in the LV-NC-shRNA and control cells as detected by western blotting (Fig. 1A and B).

APE1 silencing significantly reduces $M H C C 97-H$ cell proliferation. After infection, MHCC97-H cell proliferation rates were evaluated in the LV-APE1-shRNA, LV-NC-shRNA and negative control cells. As shown in Fig. 1C, cell proliferation was markedly decreased by knockdown of APE1 in the LV-APE1-shRNA cells $(0.2871 \pm 0.1118)$ compared to the LV-NC-shRNA $(0.5213 \pm 0.2551)$ and negative control $(0.5515 \pm 0.2659)$ cells. However, the proliferation rate was almost equal in the LV-NC-shRNA and blank control groups. 
A

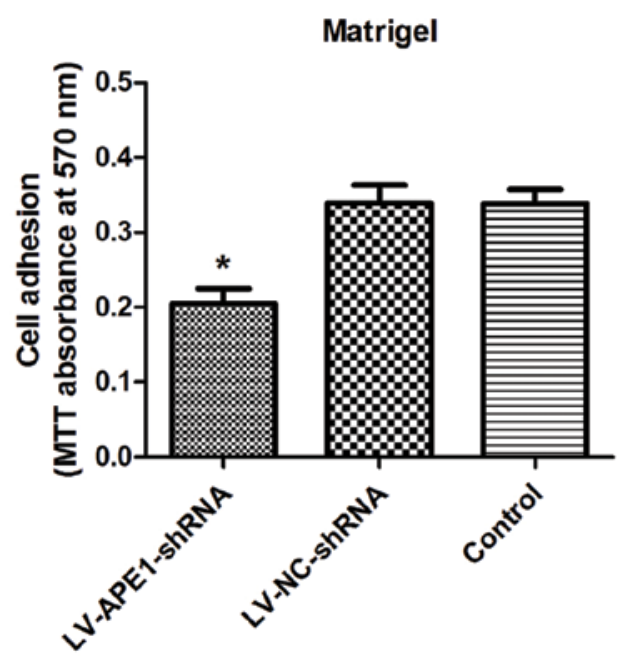

C

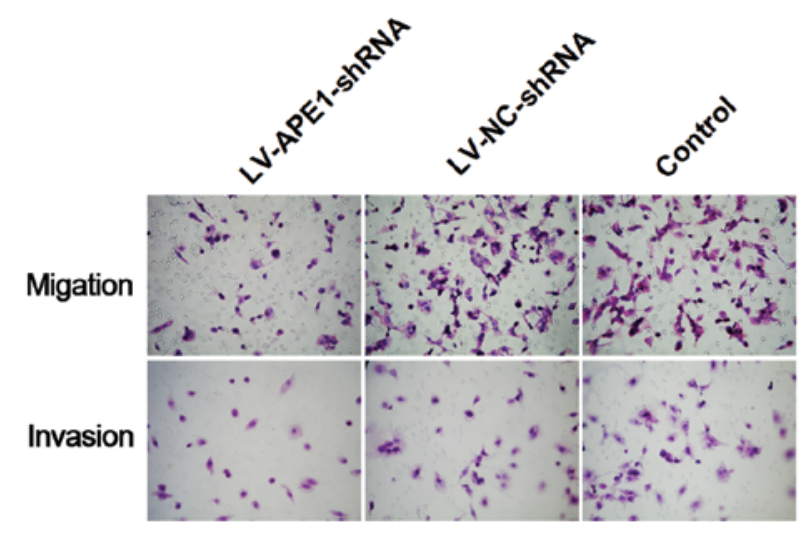

B

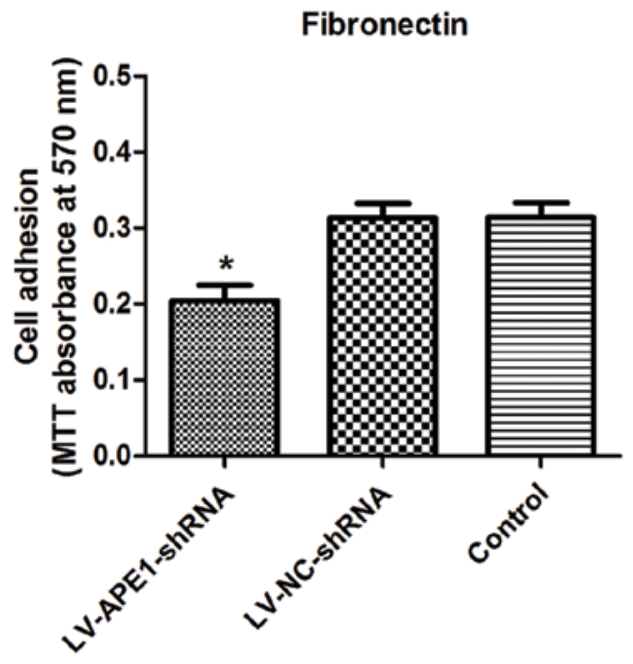

Migration and invasion

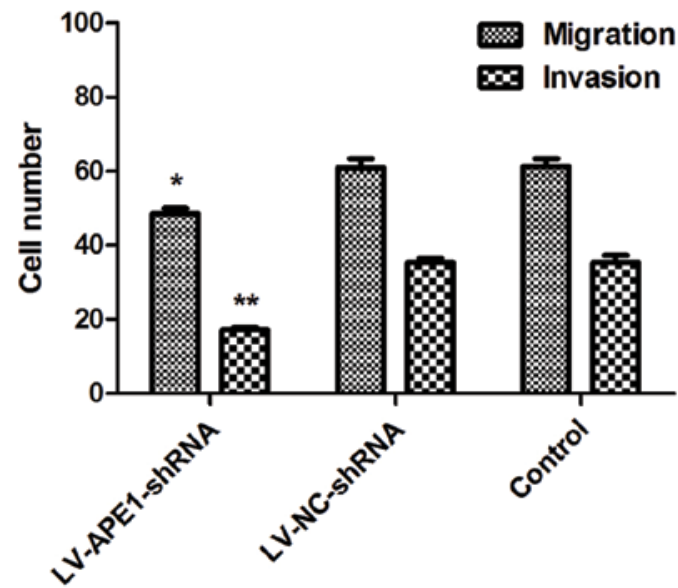

Figure 2. Silencing of APE1 decreases MHCC97-H cell migration. (A) Cell viability of the different cells in Matrigel-coated plates. (B) Cell viability of the different cells in fibronectin coated plates. (C) Cell migration and invasion assays. (D) Migration and invasion cell numbers. ${ }^{*} \mathrm{P}<0.05,{ }^{* * *} \mathrm{P}<0.01$.

APE1 silencing inhibits HCC cell adhesion ability. Cell adhesion was evaluated by cell-matrix adhesion, which was measured by MTT assay to reflect the cell adhesion ability. As shown in Fig. 2A, cell adhesion was significantly inhibited in the LV-APE1-shRNA cells compared to that in the LV-NC-shRNA and negative control cells in the Matrigel-coated plates. The data were similar with the results using FN-coated plates (Fig. 2B). Thus, silencing of APE1 significantly inhibited HCC cell adhesion ability.

APE1 silencing reduces cell invasive ability. Cell migration and invasion were investigated by Transwell assays. Cell migration was slightly decreased following the silencing of APE1 in vitro. However, cell invasion was markedly reduced in the LV-APE1-shRNA cells compared to that in the LV-NCshRNA and negative control cells (Fig. 2C). Cell counting results showed that the cell migration in the LV-APE1-shRNA cells was decreased $\sim 26 \%$ compared to the LV-NC-shRNA and negative control cells (Fig. 2D).
APE1 knockdown inhibits HCC tumor xenograft growth. APE1-knockdown MHCC97-H cells were subcutaneously injected into nude mice to investigate tumor growth in vivo. Consistent with the in vitro data presented above indicating that proliferation was significantly enhanced in cells infected with LV-NC-shRNA and markedly reduced in APE1-depleted cells, tumor growth was observed in the nude mice injected with LV-NC-shRNA MHCC97-H cells and compared to those injected with LV-APE1-shRNA MHCC97-H cells. The volume of tumors was also measured. Firstly, the volume of the tumor xenografts from the LV-APE1-shRNA group showed a marked reduction in growth rate compared to tumors obtained from the LV-NC-shRNA and negative control groups after 9 days. Furthermore, the final weight of the tumor xenografts from the LV-APE1-shRNA group was $~ 315 \%$ less than the final weight of the tumor xenografts from the LV-NC-shRNA and negative control groups (Fig. 3). The inhibition rate was $75.9 \%$ in the LV-APE1-shRNA group compared to the control groups in the final stage by measuring the weight of the xenografts (Table II). 
A

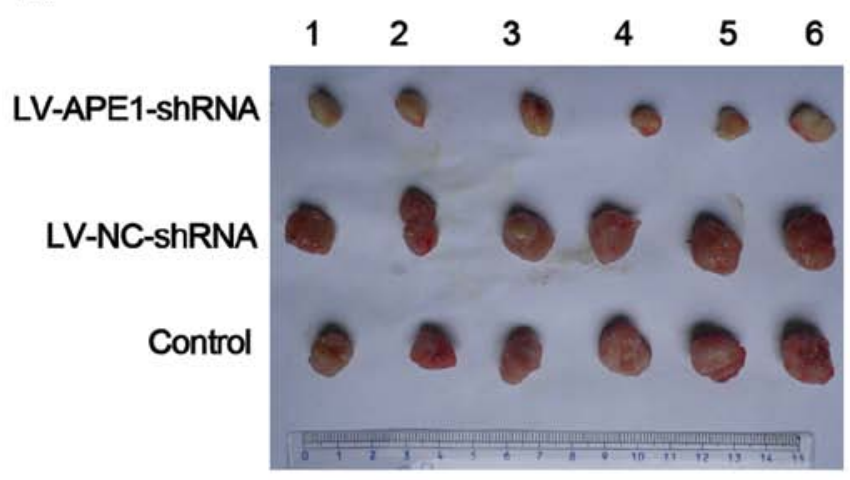

B

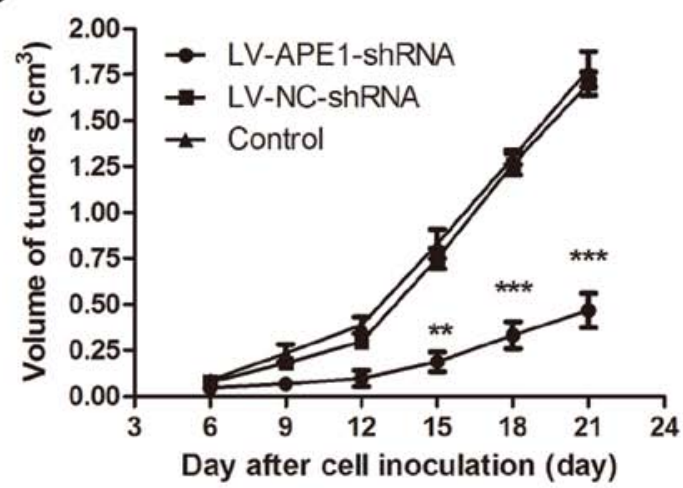

Figure 3. Silencing of APE1 inhibits tumor formation in the MHCC97-H cell-derived tumors in vivo. (A) Tumors in the different nude mouse groups injected with LV-APE1-shRNA, LV-NC-shRNA or control cells. (B) Measurement of tumor volume after inoculation from day 6 to $21 .{ }^{* *} \mathrm{P}<0.01$, ${ }^{* * *} \mathrm{P}<0.001$.

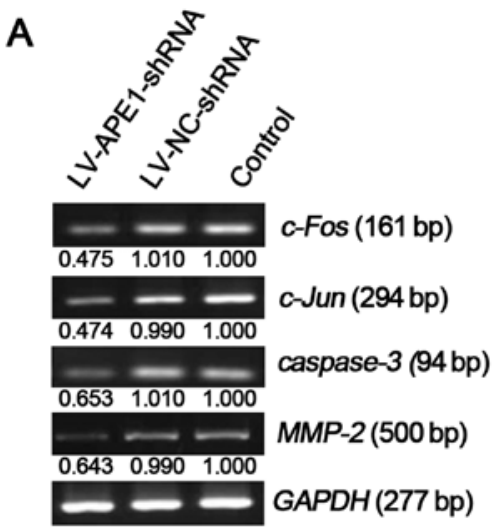

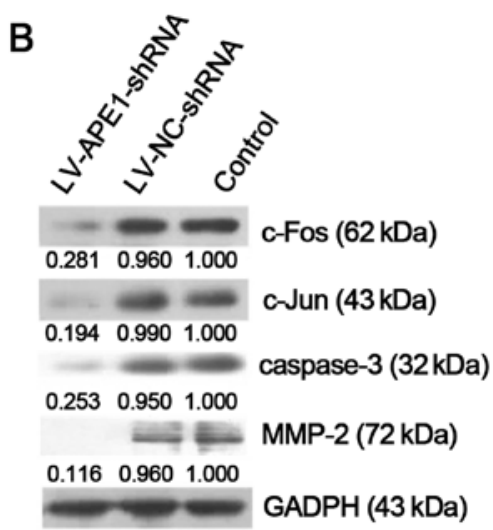

Figure 4. Expression analysis of various genes at the mRNA and protein levels. (A) mRNA expression analysis by RT-PCR. (B) Protein expression analysis by western blotting.

Table II. Silencing of APE1 inhibits tumor formation of the MHCC97-H cell-derived tumors in vivo.

\begin{tabular}{lccc}
\hline Group & $\mathrm{n}$ & $\begin{array}{c}\text { Weight of } \\
\text { tumors }(\mathrm{g})\end{array}$ & $\begin{array}{c}\text { Inhibition } \\
\text { ratio (\%) }\end{array}$ \\
\hline LV-APE1-shRNA & 6 & $0.267 \pm 0.082^{\mathrm{a}}$ & 73.772 \\
LV-NC-shRNA & 6 & $1.018 \pm 0.160$ & - \\
Control & 6 & $1.107 \pm 0.178$ & - \\
\hline
\end{tabular}

${ }^{\mathrm{a}} \mathrm{P}<0.05$

Gene and protein expression profile analysis. RNA and protein were extracted from the xenografts, and expression levels of various genes were assessed by RT-PCR and western blotting. Compared with negative control, the LV-APE1-shRNA group showed a significantly decrease in mRNA expression of c-Fos (47.580\%), c-Jun (47.402\%), caspase-3 (65.355\%) and MMP-2 (64.369\%) (Fig. 4A). These observations were consistent at the protein level, where the LV-APE1-shRNA group exhibited a significant decrease in c-Fos, c-Jun, caspase-3 and MMP-2 expression by $71.872,80.399,75.616$ and $88.393 \%$, respectively (Fig. 4B).
APE1 silencing accelerates the apoptosis of HCC cells and reduces the number of irregular mitoses. When comparing the different samples from the LV-APE1-shRNA, LV-NC-shRNA and negative control groups, we identified a positive qualitative difference caused by silencing of APE1. From the H\&E staining results, less tissue necrosis and more small cancer cell apoptotic bodies (indicating as red dye and small round body) were observed in the LV-APE1-shRNA tumors compared to the LV-NC-shRNA and negative control tumors (Fig. 5A). Furthermore, according to the pathological classification, the irregular mitosis was grouped into different types, such as tripole, asymmetrical and popcorn-like. By counting the number of irregular mitoses, we found that irregular mitotic tumor cells numbered $<5 / \mathrm{HPF}$ in the LV-APE1-shRNA group, while these numbers in the LV-NC-shRNA and negative control groups were $>5 / \mathrm{HPF}$.

Apoptosis index can be calculated by counting the apoptotic cell number using the TUNEL method. As shown in Fig. 5B, a higher number of apoptotic cells was observed as black dots in the LV-APE1-shRNA group when compared to the LV-NC-shRNA and negative control groups in the TUNEL-stained tissue samples. The apoptosis index was significantly increased in the LV-APE1-shRNA group which was almost double than that in the LV-NC-shRNA and negative control groups (Fig. 5C). 
A

LV-APE1-shRNA LV-NC-shRNA Control

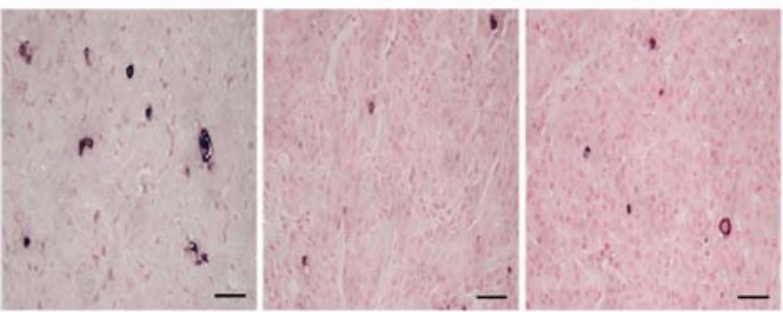

B

LV-APE1-shRNA LV-NC-shRNA Control

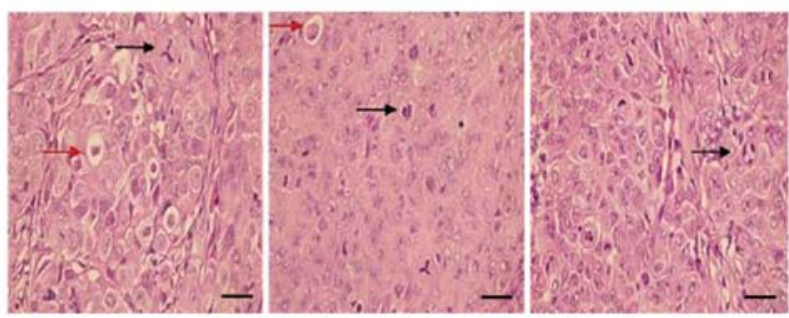

C

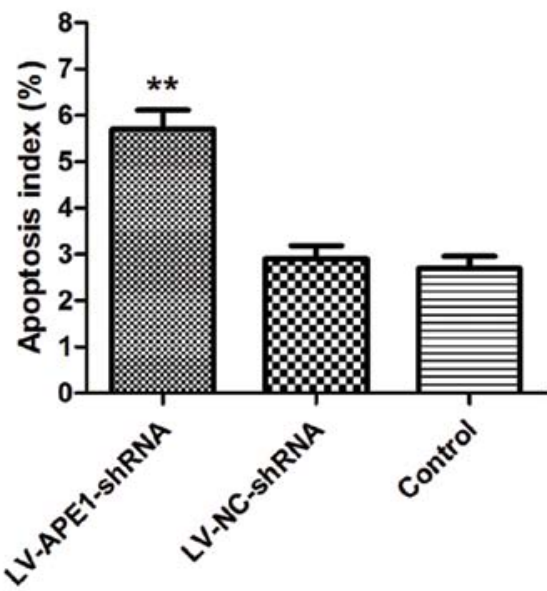

Figure 5. Immunohistological analysis of the nude mouse xenograft tumors (A) H\&E staining of tumor samples from the nude mice. (B) Analysis of apoptosis using TUNEL staining. Scale bar, $100 \mu \mathrm{m}$. (C) Apoptosis index. ${ }^{* *} \mathrm{P}<0.01$

\section{Discussion}

The poor prognosis of HCC patients is mainly due to the high frequency of late-stage cancer and metastasis at diagnosis. Despite benefits from surgical resection and a low risk of complications, only a limited proportion of HCC patients are eligible to undergo optional resection at diagnosis. Chemotherapy represents the main therapeutic option for these HCC patients, but neither single drug nor multiple drug treatments prolong the survival of late-stage HCC patients (22). In order to improve therapeutic efficacy, targeted drugs such as sorafenib are currently used in late-stage HCC patient. Sorafenib is a multikinase inhibitor that has already been approved for the treatment of advanced HCC (23). Combination of chemotherapy drugs with a targeted drug offers a novel therapeutic approach for HCC treatment. However, the adverse effects and acquisition of drug resistance of patients to sorafenib remain a problem in the clinic (24). It is necessary to explore more promising and specific targeted drugs for HCC particularly for late-stage HCC patients with metastasis.

APE1 is a multifunctional gene which regulates DNA repair and related gene expression during cell damage and cancer development. Based on our previous study, APE1 was shown to be highly expressed in poorly differentiated, capsular invasion and metastasis groups (18). In addition, we also found that APE1 protein is mainly expressed in the nuclei in normal liver tissues, while in malignant liver tissues, APE1 protein is expressed in the nucleus and cytoplasm. Such a differentiated expression pattern of APE1 indicates that it can be a potential marker for cancer diagnosis and treatment. Previously, the function of APE1 in HCC was found to be associated with cell apoptosis and resistance to radiotherapy and chemotherapy (10). Cun et al found that knockdown of APE1 increased the sensitivity of human HCC cells to radiotherapy in vitro and in vivo (12) and Zou et al demonstrated that inhibition of APE1 by a small-molecule inhibitor suppresses pancreatic cancer cell proliferation and migration (25). Moreover, inhibition of APE1 by small-molecular agents also inhibited the growth of tumor endothelium and endothelial progenitor cells (26). These results suggested an important role of APE1 in cancer and that the function of APE1 can be investigated by silencing of APE1. Lentiviral-mediated silencing of genes through RNA interference is an ideal approach that can achieve long-lasting transgene expression (27). In the present study, we selected lentiviral-mediated shRNA silencing of the APE1 gene, which can provide more stable expression compared to atopic transfection using liposome transfection.

Previous studies have shown that APE1 is a redox activator in the cell nucleus, which can activate the DNA binding activity of transcription factors associated with cancer development such as AP-1, NF- $\kappa$ B and P53 $(28,29)$. The AP-1 complex has been implicated in the transformation and progression of cancer (30). It was reported that activation of AP-1 transcription factors is frequently found in the early event of human HCC development (31). Firstly, AP-1 is comprised of heterodimers of Fos and Jun family proteins, which bind to a consensus DNA sequence that are often located in the target promoter region of genes. $c$-fos and $c$-jun are proto-genes that are important in cell cycle progression as well as proliferation (32). Secondly, altering the expression of AP-1 component proteins also affects cellular invasion (33). AP-1-regulated genes play a role in the invasive process. For example, inducible matrix metalloproteinases (MMPs) share a consensus AP-1 binding site in their promoter, which is found to be expressed in various invasive tumors (34). In addition, Fishel et al found that c-Fos can induce expression of MMP-1, MMP-2 and MMP-3 and initiate tumor progression (35). On the contrary, invasiveness of cells was significantly reduced when expression of the AP-1 component and AP-1 protein activity were inhibited (32). In the present study, we found that silencing of APE1 decreased c-Fos and MMP-2 expression, which consequently inhibited cell-matrix adhesion, cell migration and invasion. These results indicate that AP-1 is one of the downstream effectors of APE1, confirming APE1 as regulator of cancer cell proliferation and migration.

Targeted APE1 therapy was assessed in a previous study, suggesting that APE1 expression level and its deregulation in 
cells can be used as a therapeutic target for tumor radiotherapy and chemotherapy sensitivity prediction (35). Our data validated the possibility of using APE1 as a target for inhibiting tumor growth in vivo. In the LV-APE1-shRNA group, the final weight of the tumor xenografts was $\sim 315 \%$ less than that in the control group. The inhibition rate was $75.9 \%$ in the LV-APE1-shRNA group compared to the control groups in the final stage by measuring the weight of xenografts. This result is in line with previous results which found that inhibition of APE1 reduced the expression of c-Fos, resulting in a decrease in cell migration and invasion (28). Moreover, silencing of APE1 enhanced the sensitivity of HCC cells to radiotherapy (12). Therefore, APE1 as a tumor suppressor in HCC could be used in targeted therapy combined with other treatment strategies such as surgery, chemotherapy, radiotherapy and immunotherapy.

In conclusion, the present study demonstrated that APE1 silencing by shRNA suppressed the proliferation and mobility of HCC cells. Firstly, silencing of APE1 expression in HCC cells decreased cell viability and survival compared with the control cells in vitro while the cell proliferation rate in the APE1-silenced group was significantly higher than that in the control cells. In addition, APE1 was important in cell-matrix adhesion, cell migration and invasion. Silencing of APE1 expression markedly decreased cell adhesion in Matrigel or FN-coated plates. Moreover, cell migration and invasion were also decreased when APE1 expression was depleted. Finally, xenograft growth was inhibited by silencing of APE1 expression compared with that in the control groups in nude mice. Activation of protein expression levels were found to be associated with APE1 expression by western blot and RT-PCR analyses. Expression of c-Fos and c-Jun were downregulated by silencing of APE1, while expression of caspase-3 and MMP-2 were also decreased. These results suggest that APE1 is an important gene in the regulation of HCC cell proliferation and mobility by regulating related gene expression. These findings suggest that APE1 may be useful in cancer targeted drug development for inhibition of cancer metastasis.

\section{Acknowledgements}

This study was supported by the United Health and Education Research Project of Fujian Province (grant no. WKJFJ-04), the Scienctific Research Project of Education Department of Fujian Province (grant no. 2013B009), as well as the Research Fund of the Health System for Young Talents of Fujian Province (grant no. 2014-ZQN-JC-24).

\section{References}

1. Forner A, Llovet JM and Bruix J: Hepatocellular carcinoma. Lancet 379: 1245-1255, 2012.

2. Jemal A, Bray F, Center MM, Ferlay J, Ward E and Forman D: Global cancer statistics. CA Cancer J Clin 61: 69-90, 2011.

3. Tang ZY: Hepatocellular carcinoma - cause, treatment and metastasis. World J Gastroenterol 7: 445-454, 2001.

4. Bruix J and Sherman M: American association for the study of liver diseases: management of hepatocellular carcinoma: an update. Hepatology 53: 1020-1022, 2011.

5. Abbotts R and Madhusudan S: Human APendonuclease 1 (APE1) from mechanistic insights to druggable target in cancer. Cancer Treat Rev 36: 425-435, 2010.
6. Kelley MR, Cheng L, Foster R, Tritt R, Jiang J, Broshears J and Koch M: Elevated and altered expression of the multifunctional DNA base excision repair and redox enzyme Ape1/ref-1 in prostate cancer. Clin Cancer Res 7: 824-830, 2001.

7. Yoo DG, Song YJ, Cho EJ, Lee SK, Park JB, Yu JH, Lim SP, Kim JM and Jeon BH: Alteration of APE1/ref-1 expression in non-small cell lung cancer: the implications of impaired extracellular superoxide dismutase and catalase antioxidant systems. Lung Cancer 60: 277-284, 2008.

8. Xiang DB, Chen ZT, Wang D, Li MX, Xie JY, Zhang YS, Qing Y, Li ZP and Xie J: Chimeric adenoviral vector Ad5/F35-mediated APE1 siRNA enhances sensitivity of human colorectal cancer cells to radiotherapy in vitro and in vivo. Cancer Gene Ther 15: 625-635, 2008.

9. Tanner B, Grimme S, Schiffer I, Heimerdinger C, Schmidt M, Dutkowski P, Neubert S, Oesch F, Franzen A, Kölbl H, et al: Nuclear expression of apurinic/apyrimidinic endonuclease increases with progression of ovarian carcinomas. Gynecol Oncol 92: 568-577, 2004.

10. Herring CJ, West CM, Wilks DP, Davidson SE, Hunter RD, Berry P, Forster G, MacKinnon J, Rafferty JA, Elder RH, et al: Levels of the DNA repair enzyme human apurinic/apyrimidinic endonuclease (APE1, APEX, Ref-1) are associated with the intrinsic radiosensitivity of cervical cancers. Br J Cancer 78: 1128-1133, 1998

11. Wang D, Luo M and Kelley MR: Human apurinic endonuclease 1 (APE1) expression and prognostic significance in osteosarcoma: Enhanced sensitivity of osteosarcoma to DNA damaging agents using silencing RNA APE1 expression inhibition. Mol Cancer Ther 3: 679-686, 2004.

12. Cun Y, Dai N, Xiong C, Li M, Sui J, Qian C, Li Z and Wang D: Silencing of APE1 enhances sensitivity of human hepatocellular carcinoma cells to radiotherapy in vitro and in a xenograft model. PLoS One 8: e55313, 2013.

13. Tell G, Quadrifoglio F, Tiribelli C and Kelley MR: The many functions of APE1/Ref-1: not only a DNA repair enzyme. Antioxid Redox Signal 11: 601-620, 2009.

14. Bhakat KK, Mantha AK and Mitra S: Transcriptional regulatory functions of mammalian AP-endonuclease (APE1/Ref-1), an essential multifunctional protein. Antioxid Redox Signal 11: 621-638, 2009.

15. Li M and Wilson DM III: Human apurinic/apyrimidinic endonuclease 1. Antioxid Redox Signal 20: 678-707, 2014.

16. Di Maso V, Avellini C, Crocè LS, Rosso N, Quadrifoglio F, Cesaratto L, Codarin E, Bedogni G, Beltrami CA, Tell G, et al: Subcellular localization of APE1/Ref-1 in human hepatocellular carcinoma: possible prognostic significance. Mol Med 13: 89-96, 2007.

17. Zhang QH, Xiang DB, Li MX, Liao PL, Li ZP and Wang D: Expression of DNA repair gene apurinic/apyrimidinic endonuclease 1 and its correlation with the expression of mutant p53 in hepatocellular carcinoma. Chin J Dig Surg 8: 453-456, 2009.

18. Huang AM, Zheng ZH, Liu JF, Zang SB, Gao LY and Chen SP: The expression of APE1 gene and its clinical implication in hepatocellular carcinoma: a study using tissue chip assay. Zhonghua Gan Zang Bing Za Zhi 16: 542-543, 2008 (In Chinese).

19. Li YJ, Zheng ZH, Liu JF, Gao MQ and Huang AM: Construction and identification of a lentiviral vector for RNA interference of APE1 gene. J Fujian Med Univ 44: 86-90, 2010.

20. Casey RC, Oegema TR Jr, Skubitz KM, Pambuccian SE, Grindle SM and Skubitz AP: Cell membrane glycosylation mediates the adhesion, migration, and invasion of ovarian carcinoma cells. Clin Exp Metastasis 20: 143-152, 2003.

21. Fischer AH, Jacobson KA, Rose J and Zeller R: Hematoxylin and eosin staining of tissue and cell sections. CSH Prot 2008: pdb.prot4986, 2008.

22. Di Maio M, De Maio E, Perrone F, Pignata S and Daniele B: Hepatocellular carcinoma: systemic treatments. J Clin Gastroenterol 35 (Suppl 2): S109-S114, 2002.

23. Llovet JM, Ricci S, Mazzaferro V, Hilgard P, Gane E, Blanc JF, de Oliveira AC, Santoro A, Raoul JL, Forner A, et al: SHARP investigators study group: sorafenib in advanced hepatocellular carcinoma. N Engl J Med 359: 378-390, 2008.

24. Llovet JM and Bruix J: Molecular targeted therapies in hepatocellular carcinoma. Hepatology 48: 1312-1327, 2008

25. Zou GM and Maitra A: Small-molecule inhibitor of the AP endonuclease 1/REF-1 E3330 inhibits pancreatic cancer cell growth and migration. Mol Cancer Ther 7: 2012-2021, 2008. 
26. Zou GM, Karikari C, Kabe Y,Handa H, Anders RA and Maitra A: The APE-1/Ref-1 redox antagonist E3330 inhibits the growth of tumor endothelium and endothelial progenitor cells: therapeutic implications in tumor angiogenesis. J Cell Physiol 219: 209-218, 2009.

27. Morris KV and Rossi JJ: Lentiviral-mediated delivery of siRNAs for antiviral therapy. Gene Ther 13: 553-558, 2006.

28. Ando K, Hirao S, Kabe Y, Ogura Y, Sato I, Yamaguchi Y, Wada $\mathrm{T}$ and Handa H: A new APE1/Ref-1-dependent pathway leading to reduction of NF-kappaB and AP-1, and activation of their DNA-binding activity. Nucleic Acids Res 36: 4327-4336, 2008.

29. Eferl R and Wagner EF: AP-1: a double-edged sword in tumorigenesis. Nat Rev Cancer 3: 859-868, 2003.

30. Liu P, Kimmoun E, Legrand A, Sauvanet A, Degott C, Lardeux B and Bernuau D: Activation of NF-kappa B, AP-1 and STAT transcription factors is a frequent and early event in human hepatocellular carcinomas. J Hepatol 37: 63-71, 2002.
31. Yuen MF, Wu PC, Lai VC, Lau JY and Lai CL: Expression of c-Myc, c-Fos, and c-jun in hepatocellular carcinoma. Cancer 91: 106-112, 2001

32. Ozanne BW, McGarry L, Spence HJ, Johnston I, Winnie J, Meagher L and Stapleton G: Transcriptional regulation of cell invasion: AP-1 regulation of a multigenic invasion programme. Eur J Cancer 36: 1640-1648, 2000.

33. Westermarck J and Kähäri VM: Regulation of matrix metalloproteinase expression in tumor invasion. FASEB J 13: 781-792, 1999.

34. Lam E, Kilani RT, Li Y, Tredget EE and Ghahary A: Stratifin-induced matrix metalloproteinase-1 in fibroblast is mediated by c-fos and p38 mitogen-activated protein kinase activation. J Invest Dermatol 125: 230-238, 2005.

35. Fishel ML and Kelley MR: The DNA base excision repair protein Ape1/Ref-1 as a therapeutic and chemopreventive target. Mol Aspects Med 28: 375-395, 2007. 